\title{
Very High Throughput Many-Persons-To-1-Test-Kit Infectious Disease Screening
}

Hiok Nam Tay ( $\nabla$ hioknam@yahoo.com )

CANDELA Microsystems

Method Article

Keywords: COVID-19, coronavirus, screening, many persons, sample pooling, infection

Posted Date: April 28th, 2020

DOI: https://doi.org/10.21203/rs.3.pex-900/v1

License: (9) This work is licensed under a Creative Commons Attribution 4.0 International License. Read Full License 


\title{
Very High Throughput Many-Persons-To-One-Test-Kit Infectious Disease Screening By Pooling Samples
}

\author{
Hiok-Nam Tay (htay@yahoo.com )
}

\section{ABSTRACT}

\section{Background}

The 2020 COVID-19 pandemic puts tremendous pressure on hospitals and medical supplies. Nationwide lockdowns and bans on international and domestic flights fail to halt its advance nor break its exponential growth. Reportedly one quarter or more of the infected are asymptomatic but nonetheless capable of shedding the virus.

Blanket screening to isolate all infected individuals seems the only way to stop the pandemic. Unfortunately, it is not available due to practical reasons. RT-PCR tests, being very expensive, are never in sufficient capacity for screening entire population, let alone doing so continually. Besides, linear ramp-up of production is no match for the exponential growth of the disease.

No other approaches can meet the urgent need to halt the virus' advance. Vaccines are at best a year away. Antibody tests are ineffective in the initial week of infection, leaving contact tracing running behind by up to two generations of transmission. A better answer is needed to stop this pandemic and prevent future ones.

And, to exit lockdown, concrete, sound measures are needed to prevent the pandemic's return.

\section{New Many Persons To One Test Kit Methodology}

We presented a new idea--multiplier grid (MG)--in March 2020. Using a moderate number of test kits, it screens a large number of individuals to look for a small number of infected individuals. MG vastly reduces the quantity of reagents and equipment required for blanket screening as long as the infected individuals make up less than $1 \%$ of the population. The smaller the percentage, the larger the multiplier can be used by chooshing the parameters of the MG appropriately.

Now, we show how to implement the MG on existing diagnostic technologies and existing automated lab equipment in diagnostic labs.

A robust public health surveillance sytem should aim for detection and isolation before the disease carriers reach $0.01 \%$ of the population, or 1 in 10,000 . We show, using the industry standard 96-well plate for automated lab equipment (reserving first 2 wells for positive/negative controls), that the screening for $94 * 94=\underline{8,836}$ individuals can be done using $2 * 96=\underline{192}$ sets of test kits, where the multiplier is 46 , in 3 hours. For a test that costs $\$ 250 /$ kit, per person cost goes down to about $\$ 5 /$ individual. The very low cost makes periodic blanket screening (e.g. twice weekly) as community disease surveillance feasible, not to mention avoids putting pressure on material supply and equipment time.

In a population that has basic unit, for example the household in a town, or the shared cubicle in an office tower, or the cabin in a cruise ship, pooling samples from individuals that share the same basic unit to form a pre-pooled sample can boost the multiplier further. Pre-pooled samples are subject to the same workflow above to identify the basic unit that tests positive. Individual samples 
from the tested-positive basic unit are subsequently separately screened to determine who is(are) infected. The above multiplier thus becomes multiplied by the average size of the basic unit. For average household size of 4 , the final multiplier thus becomes $46 * 4=184$, and the number of individuals screened becomes $94 * 94 * 4=\underline{34,344}$, using only $2 * 96+4=\underline{196}$ sets of test kits.

\section{Applications}

MG makes blanket screening of passengers at airport possible to guarantee air travel totally COVID-19-free, to help lift bans from commercial flights. It can screen entire schools at once to ensure the first carrier to show up is isolated within a few hours. It can screen all asymptomatic section of a population to identify hidden carriers to block underground transmission. It can screen an entire factory to ensure no cluster can propagate. It can screen all restaurant and market workers in a city to make sure this group does not become a superhighway for the pathogen due to their very high frequency of close contact with clients. It can surveil for recurrence of the pandemic to effectively and speedily shut it down in one week. An when a new epidemic emerges, it can be deployed to all ports of call of a country to blanket screen all transiting people, long before mass production of specific test kits becomes availble. In many ways, it helps to return healthy security and confidence back to commerce, travel, hospitality, industry, and transportation, thus safeguarding economy security from future infectious diseases.

For a very busy international airport like the Los Angeles International Airport, with more than 10,000 arrivals an hour, up to 20 disease carriers in a day can be detected at a cost of a mere 188 test kits per hour. For a small town of 10,000, to be able to detect initial presence of and immediately isolate all disease carriers up to 20 in one day virtually guarantees to stamp out any residual trace of the disease after lockdown ends. And for a university or corporate or factory campus of 10,000 employees, MG gives the confidence to safely re-open the campus by periodically screening all its employees every three or four days until the disease is totally eradicated from the wider community.

The MG can be used in livestock farming too, to detect the first sign of infection among livestock. This can avoid mass destruction of an entire farm's livestock--which is very expensive and takes long time to recover the business from--just to prevent spread of diseases such as swine flu and avian flu.

\section{Conclusion}

MG makes a viable measure to to help stop epidemics and exit lockdown by driving down the screening cost per individual by orders of magnitude and with it the demand pressure for material supply and equipment. It can help return health security and confidence back to commerce, travel, hospitality, industry, and transportation, as well as safeguard a countrys' economic security against future infectious diseases. 


\section{Introduction to the MG Method}

\section{Background:}

In a previous paper circulated by email and a description posted online before that, this author described a cost-, time- and material-efficient method ("multiplier grid (MG) method") to perform screening to look for a few disease carriers among many individuals. The number of individuals can be 10,000 , e.g. in a small town or a university campus, or 1,000, e.g. in a school or a corporate campus.

Given the large number of individual samples involved, a very high throughput (VHT) workflow is needed to put the method to practice.

Besides, if it is to support blanket screening at an international airport that sees more than 100,000 departures a day, with some 10,000 or more an hour, it must not take too long. Here, turnaround time is also an important factor of consideration.

In many countries, the number of hospital beds ranges from 1 to 3 for every 1,000 persons. Since blanket screening should be done before patient number begins to overload the hospitals, the MG method is expected to be deployed long before disease carriers hit $0.1 \%$ of the population, or 1 in 1,000 . This means a robust public health surveillance sytem should aim for detection and isolation before the percentage hits $0.01 \%$, or 1 in 10,000 . We show, using the industry standard 96-well plate for automated lab equipment (reserving first 2 wells for positive/negative controls), that screening for $94 * 94=8,836$ individuals can be done using $2 * 96=192$ sets of test kits, achieving a multiplier of 46 . For a test that costs $\$ 250 /$ kit, that goes down to about $\$ 5 /$ individual, making periodic blanket screening as disease surveillance acceptable in terms of cost, not to mention avoiding putting pressure on material supply and equipment time.

For a very busy international airport like the Los Angeles International Airport, with more than 10,000 arrivals an hour, it means a mere 192 test kits per hour suffices to detect up to 20 disease carriers in a day, which is expected some 2 months after outbreak begins in some foreign country of an infectious disease with incubation period of 5 days. For a small town with that size of population that has just emerged from lockdown, to be able to detect presence of and immediately isolate up to 20 infected persons in one day virtually guarantees to block return of the disease. And for a corporate campus of 10,000 employees, to be able to safely re-open the campus after lockdown depends on notonly on blanket screening but also on periodic screening of its entire campus population every three or four days until the disease is totally banished from the wider community.

\section{Very High Throughput (VHT) MG Workflow:}

By way of example, this paper presents a very high throughput (VHT) MG workflow (Fig. 1) based on commercially available high throughput (HT) equipment already in use in the medical diagnostics industry. It can be easily modified to suit different parameter values ( $\mathrm{N}, \mathrm{P}$, plate well count, etc.) and optimization goals (turnaround time, cost, reagent quantities, intrinsic clustering \& hierarchies within population, etc.). See final section in this paper, Suggested Modifications \& Optimizations.

The example below is a two $(\mathrm{P}=2)$ dimensional multiplier grid having axis size $\mathrm{N}=94$, capable of screening $94 * 94=8,836$ samples in each run in under three(3) hours (including 1 hour for qPCR 
reactions). Extension to higher dimension and/or different $\mathrm{N}$ values and/or 384-well plates will become self-evident.

To find out which one of the $94 * 94=8,836$ input samples carries a pathogen, the MG method proceeds as follows, consuming merely $2 * 96=192$ sets of reagents, reactions and equipment time instead of 8,836 sets. Multiplying factor is 46 times. Thus, a 46-to-1 test methodology.

For VHT workflow, lay input samples out across 94 industry-standard 96-well plates, the 96 wells laid out in 12 columns (labeled columns 1 to 12 from left to right) by 8 rows (labeled rows A to $\mathrm{H}$ from top to bottom) (Fig. 2). Leave the first two wells-well A1 \& A2-empty for adding positive and negative controls just before reactions. Label the plates a3 through h12, like the wells--except lowercase roman letters are used to distinguish plate labels from well labels--, for plate-to-well pooling in Step 2 below. Altogether there are $94 * 94=8,836$ input samples, each $\underline{\underline{1 \mathrm{~mL}}}$.

1. First, the MG workflow tries to find out if and where the pathogen exists among the 94 plates of input samples. To do so, it needs only 94 sets of reagents and reactions and equipment time. It will collect a fraction of the sample fluid in each well—called aliquotfrom plate a3 - the first plate-, add up aliquots from all 94 wells in the same input sample plate to form a pooled sample, concentrate it, purify it, and send for reaction (Fig. 1). Likewise for plate a4. Next, plate a5. And so on, until plate h12.

2. In practice, high throughput (HT) machines will run reactions on all 94 pools of samples concurrently in a plate of 96 wells ("Set 1 pooled sample plate"), with wells A3 through H12 holding the pooled samples from input sampling plates a3 through h12, respectively, while wells A1 and A2 holding positive/negative controls (Fig. 2, left half).

3. If all come up empty (aka negative), then that's it-there's no pathogen, all input samples are in the clear, and we move on.

4. But if there is one (or more) input sample that has the pathogen, one (or more) of the plates will turn up positive from the reactions. Then the method continues as follows.

5. Get a new 96-well plate. Leave the first two wells empty for positive/negative controls. Transfer aliquots from only the tested-positive input sample plate(s), with wells A3 to H12 receiving aliquots from the respective same-label wells of those plates.

6. In fast turnaround practice, setting up this new plate only after results from reactions on the Set 1 pooled sample plate are known adds unacceptable delay. In this situation, this new plate is set up and sent for reactions concurrently with the Set 1 pooled sample plate. In order to do so, instead of selecting only tested-positive input sample plates to pool aliquots from their wells, add up aliquots from all (94) same wells across all (94) input sample plates to place into each well of the new plate ("Set 2 pooled sample plate") (Fig. 2, right half).

7. Take this Set 2 pooled sample plate through concentration, purification and reactions (Fig. 1). If none turns out positive, something is wrong with either this set of reactions or the previous one. Otherwise, at least one out of 94 will turn out positive. 
8. Say exactly one plate turns positive, say plate e7, and exactly one reaction in the second set of reactions turns positive, say well D11. Then the pathogen has been found in input sample plate e7, its well D11. In this scenario, we have located the pathogen using $96 * 2=\underline{192 \text { sets }}$ of reagents and reactions.

9. Alternatively, say two input sample plates turn positive, say plate e7 and h2, and two reactions in the second set of reactions turn positive, say well D11 and G8. Then the pathogen has been found those wells in those input sample plates, in total 4 wells: (1) input sample plate e7 in its wells D11 and G8, and (2) input sample plate h2 in its wells D11 and G8. Now take four aliquots from those wells from those two input sample plates and send for reactions, the third time now. It may turn out that all four test positive, or three of them, or two of them, but not just one. In this scenario, we have located the pathogen using $96 * 2+$ $4=\underline{196 \text { sets of reagents and reactions. }}$.

The concentrating and purifying steps are looked into below.

\section{Concentrating and Purifying Pooled Samples:}

We are given the following:

1. At the above Steps 1 to 2 and 6, two pooled sample plates are produced-Set 1 and Set 2 pooled sample plates. Pooling 94 aliquots—250uL each—results in fairly large volume, almost $25 \mathrm{~mL}$ - far more than can be held in a well—, and 94 of them.

2. Sample purification, here nucleic acid (RNA/DNA) extraction, using commercial kit, can only take in a small volume, 400uL for one commercial extraction kit ("sample volume”). At the end of extraction, $20 \mathrm{uL}$ is the smallest volume ("elution volume") that is produced.

3. And finally, out of the elution volume, only $\underline{\underline{\mathrm{uL}}}$ will go into each reaction.

4. Two reactions will be performed per pooled sample. Test is positive if either turns positive.

How to concentrate 2 times 94 pools of nearly $25 \mathrm{~mL} / \mathrm{pool}$ down to $400 \mathrm{uL} / \mathrm{pool}$ (about 60X) in a high throughput manner is the problem being addressed in this paper in order to make manyto-1 test methodology practical. The workflow must avoid human errors or cross contamination between samples or sample pools. This is the subject matter of the next section.

After concentrating the Set 1 and Set 2 sample pools, each set is laid out in 94 portions of 400uL/portion across 94 wells in a final pooled sample plate of 96 wells (wells A1 and A2 to be taken up by positive and negative controls). The plates are now ready to go for purification.

In sample purification, the two sets of 94 concentrates each go through conventional nucleic acid extraction to purify the pooled samples, using commercial high-throughput extractor machines and commercial high-throughput virus nucleic acid isolation kits.

Now, eluates (20uL each) from the purication step in two plates of 96 wells are ready for reactions. From each well, $5 \mathrm{uL}$ will be added to reagents for one reaction and another $5 \mathrm{uL}$ for a second reaction. 


\section{Turning $N^{\wedge} 2$ Input Samples Into 2*N Pooled Sample Concentrates: A Manual}

Summary: To support multiple persons to one test kit (virus extraction and qPCR), below is an outline of method and equipment to turn $\mathrm{N}^{\wedge} 2$ input samples (e.g swab in VTM liquid, OR sputum) into $2 * \mathrm{~N}$ pooled samples. As an example of a $2 \mathrm{D}$ multiplier grid with $\mathrm{N}=94$, input samples from 94 plates of 96 wells are pooled into 94 Set 1 canisters* (pools) and 94 Set 2 canisters* (pools). (It may be easily extended to 384-well plates for $\mathrm{N}=382$.) The first 2 wells-wells A1 and A2 -in each plate are empty. Each input sample plate pools aliquots from all its 94 wells into one and only one canister of Set 1, whereas the same wells in all plate across all 94 input sample plates pool aliquots into one and only one canister of Set 2.

From each input sample (1 $\underline{\underline{\mathrm{mL}}})$, a $\underline{\underline{250 u L}}$ (i.e. 1/4) aliquot thus goes into a Set 1 canister, and a second 250uL aliquot into a Set 2 canister. Each Set 1 pool (canister) collects 94 aliquots from all input samples of the corresponding plate (Fig. 2, left half), and each Set 2 pool (canister) collects 94 aliquots from the corresponding wells across all 94 input sample plates (Fig. 2, right half).

Next, the sample fluid pools are concentrated (e.g. by ultrafiltration in $15 \mathrm{~mL}$ microconcentrator using centrifuge) into $\underline{\underline{400 \mathrm{uL}}}$ or less, to be input to $\underline{\underline{\mathrm{RNA}} \text { extraction }}$ to produce elution in $\underline{\underline{20 \mathrm{uL}}}$, from which $\underline{\underline{5 L L}}$ (i.e. 1/4) will go into qRT-PCR analysis.

* Each canister of the multiplier grid is first represented by a pair of $15 \mathrm{~mL}$ collection tubes, next by one microconcentrator, and finally by one $400 \mathrm{uL}$ pooled sample concentrate in a 96 -well plate, ready to go into the next stage, RNA extraction.

Equipment: (Prices are indications only and provided only to aid preliminary budgeting.)

- $\quad$ 2x (semi-)automated 96-channel pipette. ( US\$10k EA used, \$20k EA new.) (Total \$20k used; \$40k new.)

- 8x (or 4x) adjustable spacing multi-channel electronic pipette (e.g. Voyager[1,250uL; 4ch:9 33mm; 6ch:9 19.8mm, ( \$2,000 EA); 8ch:9 14mm], Rainin XLS[8ch: 19mm]). https://www.integra-biosciences.com/global/en/electronic-pipettes/voyager To transfer intermediate sample fluid pools from $2.4 \mathrm{~mL}$ deep well plates to $15 \mathrm{~mL}$ collection tubes. (\$16k total)

- 8x centrifuges, with rotor and adaptor to accept 50mL conical end tubes. E.g. Eppendorf 5910R, can hold $4 * 6=24$ such micronconcentrators at once. 4 units can centrifuge 96 micronconcentrators simultaneously. ( US\$15k EA. \$120k total.)

Consumables: (Prices are indications only and provided only to aid preliminary budgeting.)

- Microconcentrator (e.g. Amicon Ultra-15 [15mL, Ø29.7mm, 121mm](Case of 96 for $\$ 1,216$ ) or Centricon Plus-20 [20mL] (£300/8pcs), Macroprep[20mL], VivaSpin 20[20mL] (pack of 48 for \$545)). https://www.sigmaaldrich.com/life-science/protein-samplepreparation/protein-concentration/amicon-ultra-centrifugal-filters/15-ml.html. https://www.sigmaaldrich.com/life-science/protein-sample-preparation/proteinconcentration/non-clinical-samples/centriprep.html https://www.fishersci.com/shop/products/emd-millipore-amicon-ultra-15-centrifugal-filterunits-15/p-4902700. 
https://www.vivaproducts.com/vivaspin-centrifugal-concentrators/vivaspin-20.html. To filter out excess fluid from sample pools. Total \$2,432.

- 12x 96-well deep well plates of 2.4mL wells (Eppendorf DWP 96/2000, 44.1mm deep), (5x PK/4 for \$140). https://www.pipette.com/EppendorfDWP?sbr=5064\&sbn=Eppendorf \%20Deepwell\%20Plates\%20(DWP) Total \$140.

- $2 * 94 \times 15 \mathrm{~mL}$ tubes $(\varnothing 17 \mathrm{~mm}$ )(Falcon conical centrifuge tubes, \$316/500). As collection tubes. Total \$316.

- 94x 96-well deep well plates (for sputums) (5x PK/4 for \$130) https://www.pipette.com/EppendorfDWP?sbr=5064\&sbn=Eppendorf\%20Deepwell \%20Plates\%20(DWP), total \$650, OR

- 94x 96-well racks (to hold swab VTM tubes), for input samples.

- 94x 96-to-1 adapters to pool from 96 pipette tips into a 25ml volume. DIY from tubes \& 3d printer. See last section on 96-to-1 adapter design.

\section{Input Samples \& Plate Labeling: Workflow}

Arrange $94 \wedge 2$ input samples into 94 input sample plates of 96 wells-arrayed in 12 columns by 8 rows - each, taking up 94 wells in each plate leaving out the first 2 wells (labeled A1 and A2) for negative $\&$ positive controls.

(The wells in each plate already come labeled rows A to $\mathrm{H}$ from top to bottom, and columns 1 to 12 from left to right. E.g. well B5 is in second row and 5th column.)

Get a new 96-well plate as a Set 1 pooled sample plate.

Label the 94 input sample plates uniquely like how wells are labeled, excluding labels A1 and A2 (for the first two wells, which are used for positive and negative controls), because in the next step each input sample plate will pool aliquots from all but two control wells into the well that takes the same label in the Set 1 pooled sample plate. Use lower case roman alphabets to differentiate from well labels, which are in upper case.

Get another new 96-well plate as a Set 2 pooled sample plate.

Record in a computer or on a sheet of paper the label of the input sample plate (e.g. plate\#c4) and the label of the well (e.g. well\#G7) that each sample goes into together with the information that identifies the source of the sample (e.g. serial number of the individual).

When, for example, pooled sample C4 in the Set 1 final sample pool plate and pooled sample G7 in the Set 2 final sample pool plate test positive in qRT-PCR, the individual who produced the input sample in plate\#c4, well\#G7 is a suspected carrier.

\section{Pooling Into Set 1 (the X Axis) Canisters: Workflow}

Take an input sample plate. Using a first 96-channel pipette, aspirate 250uL of sample fluid per well from all 94 sample wells of the input sample plate. Slide the input sample plate away.

Get a pair of new $15 \mathrm{~mL}$ tubes as collection tubes. Label them same as the input sample plate. 
Release all that 250uL from each tip, all tips, into one pool split between the two collection tubes. (96-to-12 adaptor for large collection tube is described below.) (Fig. 3)

Take a new microconcentrator (e.g. Amicon Ultra-15 [15mL, 29.7mm diameter]). Label it the same as the collection tubes. Transfer the pool to the microconcentrator and centrifuge to get the final volume below 400uL, concentrating by about 60x. (Fig. 5, top half)

Do the above for all 94 input sample plates to produce 94 pools. Each pool is about $25 \mathrm{~mL}$ in volume.

Finally, pipette from the microconcentrator into the Set 1 pooled sample plate, into a well labeled same as the microconcentrator. (Fig. 5, top half, last step)

It takes twice per pool to complete concentrating the $25 \mathrm{~mL}$ volume using $15 \mathrm{~mL}$ microconcentrators, e.g. Amicon Ultra-15. First, take one $15 \mathrm{~mL}$ collection tube from each one of the 94 pools, transfer to 94 microncentrator, label them like the respective collection tubes, close the caps, and centrifuge them in 5 centrifuges for 10 to 15 mins till only about $1 \mathrm{~mL}$ of retentate is left in the filter. Dispose of the filtrates. Next, transfer the remaining sample fluids from the remaining $15 \mathrm{~mL}$ tubes to the microconcentrators, matching labels between the collection tubes with the respective microconcentrators. Close the caps, and centrifuge again until reaching the final volume, $400 \mathrm{uL}$.

Pooling for Set 1 takes less time than sorting for Set 2 (described next) that runs concurrently, so does not factor into total time, unless the centrifuges are shared between Set 1 and Set 2 workflows, saving the cost of 4 centrifuges $(\sim 60 \mathrm{k})$, in which case expect to add 30 mins to the total sample pooling time.

Note : This concentration step may be unnecessary in screening sputum samples for SARS-CoV-2 because only $4 \mathrm{uL}$ of sputum sample needs be pipetted from each sample tube, because in most cases and most days after onset of symptoms the RNA copies/mL in sputum is 3 to 4 orders of magnitude higher than nasal/oral swabs, as shown in Figure 2 of Woelfel et al.(2020), MedRxiv, doi:10.1101/2020.03.05.20030502. Even in the few cases and days where this isn't, the RNA copy number per $\mathrm{mL}$ of sputum is greater than 6,000 . It therefore appears that a much smaller aliquot of the sputum sample suffices, and the extra costs and time used on the centrifuge may be saved. Additionally, with only a small aliquot of $4 \mathrm{uL}$ to pool from the same well in 94 sample plates, forming a pool of about 386uL, one $2.4 \mathrm{~mL}$ deep well plate in the below section on sorting into Set 2 canisters suffices to hold all 94 pools without need for the two $15 \mathrm{~mL}$ tubes and the workers to manually pipette from the twelve(12) $2.4 \mathrm{~mL}$ deep well plates to the 94 pairs of $15 \mathrm{~mL}$ tubes, as well as the equal number of 6-channel adjustable electronic pipettes. And one 500uL well plate can replace the twelve deep well plates.

\section{Pooling Into Set 2 (the Y Axis) Canisters: Workflow (1 hour)}

Using a second 96-channel pipette, pipette from the 94 input sample plates to twelve(12) 96-well plates of $2.4 \mathrm{~mL}$ wells (intermediate pooling plates) (leaving the first 2 wells empty). Every 8 input sample plates pool 250uL each into 1 intermediate pooling plate. Each well but wells A1 and A2 in the intermediate pooling plate draws from same-labelled wells from those 8 input sample plate.

(Fig. 4A). 
Next, for all 94 wells of the Set 2 pooled sample plate but wells A1 and A2, each well pools from the same-label wells across all twelve(12), as follows.

As soon as four(4) of the intermediate pooling plates are ready, the final step of pooling as describe below can begin with a team of four(4) workers. The waiting time is the time it takes to pipette from $4 * 8=32$ input sample plates to 4 intermediate pooling plates using just one 96-channel pippette. Taking into account $\sim 8$ seconds to setup each intermediate pooling plate in the pipette, and $\sim 8$ seconds for each input sampling plate, and $\sim 10$ seconds to pipette from one plate to the other, total waiting time is around $4 * 8+32 * 8+32 * 10=608$ seconds, or about 10 mins.

Before it begins, divide each intermediate pooling plate into 2-by-8 sections (named 1A, 1B; $2 \mathrm{~A}$, $2 \mathrm{~B} ; \ldots ; 8 \mathrm{~A}, 8 \mathrm{~B}$ ) of $6-$ by-1 wells each. Arrange and label 94 pieces of new $15 \mathrm{~mL}$ collection tubes in like manner to the $\underline{\underline{94} \text { input sample wells }}$ of the 96 -well input sample plates.

And, label 94 microconcentrators to match the respective $15 \mathrm{~mL}$ collection tubes.

First, pool from these 12 intermediate pooling plates to 94 pairs of $15 \mathrm{~mL}$ collection tubes as final pools as follows. (Fig. 4B)

1. Using a 6-channel adjustable electronic pipette (1250uL volume limit), transfer sample fluids of the intermediate pooling plate section-by-section into the $15 \mathrm{~mL}$ collection tubes.

To speed things up, divide up the transfer task between half (or quarter) as many workers as there are sections, i.e. 8 (or 4) workers using 8(or 4) adjustable spacing 6-channel pipettes, e.g. Worker 1 takes sections $1 \mathrm{~A}$ and $1 \mathrm{~B}$ (or $1 \mathrm{~A}, 1 \mathrm{~B}, 2 \mathrm{~A}, 2 \mathrm{~B}$ ); Worker 2 takes sections $2 \mathrm{~A}$ and 2B (or 3A, 3B, 4A, 4B); ... ; and Worker 8 (or 4) takes sections 8A and 8B (or 7A, 7B, 8A, $8 B$ ), for total 8 (or 4 ) workers.

2. After working through 6 intermediate pooling plates, move out the $15 \mathrm{~mL}$ collection tubes, and repeat for a $2^{\text {nd }}$ set of $15 \mathrm{~mL}$ collection tubes.

Based on 10 seconds for each pipette transfer, total time comes to about 10 seconds times 2 transfers per deep well times 2 (or 4) sections times 12 intermediate pooling plates, or 480 (or 960) seconds, or $\underline{8 \text { (or 16) minutes. }}$

3. Empty the $1^{\text {st }}$ set of $9415 \mathrm{~mL}$ collection tubes into 94 new microconcentrators (e.g. Amicon Ultra-15 [15mL]). Spin as many of these microconcentrators as the available centrifuge(s) can hold in the centrifuge(s) for $\underline{12 \mathrm{mins}}$ each time until retentate shrinks to $1 \mathrm{~mL}$.

With enough centrifuges, all 94 microconcentrators can be centrifuged into one go. Takes 15 mins including time to load.

4. Empty the $2^{\text {nd }}$ set of $15 \mathrm{~mL}$ collection tubes into the respective corresponding 94 microconcentrators, making sure labels between them match up.

Centrifuge all the way down to 400uL in $\sim 15$ mins.

Total time taken is nearly an hour.

Note : Such high throughput concentration becomes unnecessary where turnaround time isn't critical, becoming 5 hours. In earlier section, The Very High Throughput (VHT) MG Workflow, Step 5 replaces Step 6(short turnaround time practice) in such situation, where only one input 
sample plate that have tested positive in Set 1 pools will go for Set 2 test, or if a handful of input sample plates tested positive then only this handful of input sample plates pool together, same label wells pooling together, to go for Set 2 test.

\section{6-to-1 Adapter design: (Fig. 6)}

\section{Equipment:}

- $\quad 3 d$ printer. $(\sim 300)$.

\section{Consummables:}

- 3x 100mm long, $17 \mathrm{~mm}$ diameter $14 \mathrm{~mL}$ capacity round bottom plastic test tubes. (E.g. Falcon Round-Bottom Polypropylene Tubes, https://www.fishersci.com/shop/products/falcon-round-bottom-polypropylene-tubes-7/p196968)

- 1x 75mm long, $12 \mathrm{~mm}$ diameter $5 \mathrm{~mL}$ capacity round bottom plastic test tube. (E.g. Falcon Round-Bottom Polypropylene Tubes, https://www.fishersci.com/shop/products/falconround-bottom-polypropylene-tubes-7/p-196968) (Note. Flat bottom tube would be preferable, as shown in Fig. 6, but Falcon does not seem to have them.)

- Enough PLA filament spools to make as many adapters as required.

To pool the drips from 12 by 8 pipette tips spaced $9 \mathrm{~mm}$ center-to-center safely and not causing contamination nor introduce foreign substance into the sample pool, build a raft-like parallel array of troughs made out of lab quality plastic test tubes to guide the drips into two troughs placed closed-end to closed-end, also made of plastic test tubes, that flow into two $15 \mathrm{~mL}$ tubes held in place at opposite ends of the end-to-end troughs. Below procedures describes the design.

Take 3 pieces of $100 \mathrm{~mm}$ long, $17 \mathrm{~mm}$ diameter, $14 \mathrm{~mL}$ capacity round bottom plastic test tubes. Using hot-knife cutter, slice them down the middle into 6 halves. Lay the 6 half tubes down column wise, $18 \mathrm{~mm}$ centerline to centerline like a raft-twice the $9 \mathrm{~mm}$ pipette tip center to tip center distance-, their (previously) closed ends in the back, their (previously) open ends in the front, and concave side up to receive drips from the pipettes.

Using 3d printer, make a support frame design to rigidly hold the 6 half tubes at that $18 \mathrm{~mm}$ centerline-to-centerline spacing, with the closed ends held a little higher (say $3 \mathrm{~mm}$ ) than the open ends, so that the drips of sample fluids will flow down the half tubes towards the open ends.

Take 1 piece of $75 \mathrm{~mm}$ long, $12 \mathrm{~mm}$ diameter $5 \mathrm{~mL}$ capacity round bottom plastic test tube. Slice it down the middle into 2 halves. Place them closed end to closed end, with open ends away from each other, concave side up right, under the open ends of the raft of 6 half tubes to receive the pipetted sample fluid flowing down the raft.

With the 3d printer, extend the support frame design to hold this 2 halves in place, with the open ends slightly below the level of the closed ends so that sample fluid will flow towards the open ends. 
Finally, place two $15 \mathrm{~mL}$ new collection tubes under the two open ends to collect equal amount of sample fluids. Hold them slightly tilted towards the open ends in such a way that flows from the open ends will flow down the inside walls of the $15 \mathrm{~mL}$ collection tubes without splashing.

Now, extend the support frame design to hold this pair of $15 \mathrm{~mL}$ collection tubes firmly in place.

Finally, extend the support frame design to register its base in the right place in the pipetter station so that the adapter is positioned to receive the array of 12 by 8 pipette tips correctly.

After releasing sample fluids into the adapter, use the same pipette tips to aspirate a small amount of suitable solvent (e.g. water) and pipette it down the adapter to flush residual sample fluid out to the pair of $15 \mathrm{~mL}$ collection tubes.

\section{Extracting Virus DNA/RNA From Pooled Sample Concentrates [15m]}

\section{Background:}

After concentrating the Set 1 and Set 2 sample pools, each set is arranged in 94 portions of 400uL/portion across 94 wells in a final pooled sample plate of 96 wells (wells A1 and A2 to be taken up by positive and negative controls).

Each well of the Set 1 pooled sample plate has received aliquots from all wells (except wells A1 \& A2) of the input sample plate of the same label as the well.

Each well of the Set 2 pooled sample plate has received aliquots from all wells of the same label across all 94 input sample plates.

Organized this way, when a Set 1 pooled sample tests positive and so does a Set 2 pooled sample (e.g. Set 1 pooled sample B5 and Set 2 pooled sample G11), then the input sample in the input sample plate that corresponds to the Set 1 pooled sample (here, Set 1 pooled sample B5) and the individual input sample well that corresponds to the Set 2 pooled sample (here, Set 2 pooled sample G11), in this example input sample G11 in input sample plate b5, is identified.

\section{Extractor \& Nucleic Acid Isolation Kits:}

Before sending for analytic tests, the two sets of 94 concentrates each, each concentrate in 400uL, will go through conventional nucleic acid extraction to purify the pooled samples, using commercial high-throughput extractor machines and commercial high-throughput virus nucleic acid isolation kits.

The isolation kits are based on magnetic beads technology, which is able to produce a smaller elution volume (MagMax: 20uL) to achieve higher ratios of concentration than using filter-based silica column technologies. This is especially important where the nucleic acid (RNA or DNA) copy number per $\mathrm{mL}$ of the imput sample fluid is very low (e.g. 50 copies $/ \mathrm{mL}$, like in nasopharyngeal swab of COVID-19 patients in the $2^{\text {nd }}$ week after onset of symptoms [Woelfel 2020]).

Where the analytic test is qPCR test, because typically only $5 \mathrm{uL}(1 / 4 \mathrm{of} 20 \mathrm{uL}$, the elution volume) of sample fluid goes into a reaction with reagents, the copy number in the reaction goes down by factor of 4 here. Since we already dropped copy number by a factor of 4 when we took only 250uL ( $1 / 4$ of $1 \mathrm{~mL}$ ) of input sample fluid to pool into each sample pool, only 1/16 of the original copy number goes into the reaction, which is 50/16 3 DNA/RNA copies on average. This barely 
suffices to test positive from at least one reaction unless two reactions are performed for every pooled sample.

Below listed are the extractors and nucleic acid isolation kits that appear to be suitable. They are not meant to be exhaustive, as there are certainly other manufacturers and isolation kits available, and at various levels of efficacies, e.g. recovery rate, flexibility of protocols, and price points, to consider.

Prices are indications only and provided only to aid preliminary budgeting.

\section{Equipment :}

- 2x QIAsymphony SP extractor machine. 96 wells. 2 and $4 \mathrm{~mL}$ (fill to 4.8mL). Protocol customizable by Qiagen only. (from €85,000 x 2). OR

- 2x KingFisher Flex 96 Magnetic Particle Processor. 96 wells. 1mL. Protocol customizable by user. ( $\$ 100,000 \times 2)$ https://www.youtube.com/watch?v=cace7rRTIww . https://www.youtube.com/watch?v= cPSQZ Bqck .

\section{Consumable :}

- QIAsymphony DSP Viral/Pathogen Kit. Magnetic beads technology. Elution volume min

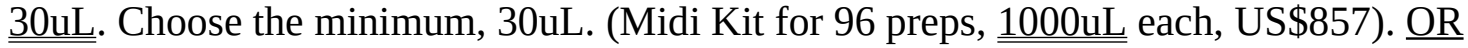

- MagMax 96 Viral/Pathogen Nucleic Acid Isolation Kit. Magnetic beads technology. Sample volume max $\underline{\underline{400 u L} .}$ Elution volume min 20uL. (Price???)

\section{Suggested Modifications \& Optimizations}

The VHT MG workflow can be easily modified to suit different parameter values (N, P, plate well count, etc.) and optimization goals (turnaround time, cost, reagent quantities, $\underline{\text { intrinsic clustering \& }}$ hierarchies within population, etc.).

\section{Intrinsic Clustering \& Its Basic Unit:}

There are populations (e.g. army camp, dormitories, condominium complex, office tower, cruise ship, school, shopping mall) where there are intrinsic clustering of people, whereby there is far more shared touched surfaces and/or breathing space between individuals belonging to the same cluster than between individuals from different clusters. Such populations tend to have a basic unit of clustering, and the population builds up hierarchically from that basic unit.

For example,

1. in a cruise ship, several people share a cabin, and the cabin is the basic unit.

2. For an office tower, the basic unit can be people working within a department. For a shopping mall, the basic unit can be workers within the same shop.

3. For schools, the basic unit is the pupils who use the same classroom as well as their teachers.

4. For a condominium complex, the basic unit is the household living in each unit.

For such populations, instead of the input sample plate assigning each individual's sample to one well, we can pool their input samples by the basic units before assigning to wells in the input 
sample plate. When a input sample well's test results turn positive, input samples from members of the corresponding basic unit are then tested individually.

Members of the same basic unit are more likely to be cross infected. Rather than scattering multiple likely cross infected samples from the same basic unit across multiple wells, it is more efficient to lump them all in one well so that other wells are able to uniquely identify other infected basic units.

There is also the benefit that the cohort size is multiplied by the average size of the basic unit. For example, for a town of $94 * 94=8,836$ households, with average household size of four individuals, the number of individuals being screened becomes $4 * 8,836=35,344$, using additional 4 more test kits on average to identify the infected members of one infected household when the household tests positive .

And for departing flights, it is more likely that individuals who carry the same infectious disease know each other before taking the flight book seats in the same row of an airplane than any two random individuals. Pooling samples from seats in the same row into a single well on an input sampling plate will therefore be more efficient than having their samples scattered across different wells.

\section{Intrinsic Clustering \& Its Hierachies:}

In a highrise residential tower, dwelling units are divided into floors, and same numbered units on different floors share the same sewage pipes and ventilation pipes that could possibly transmit disease. It is therefore more likely that individuals from same numbered units from different floors cross infect between one another than any two random individuals from the same residential tower. It would be more efficient to assign individuals sharing the same unit number to the same input sample plate than randomly, or to the same well number across different input sample plates than randomly.

Likewise, on a ship, it is more likely that individuals from cabins that share the same facilities cross infect between one another than between any two random individuals on the ship. Assigning such individuals to the same input sampling plate will be more efficient in the usage of the Set 1 pooled samples' processing than otherwise. Same goes for an office tower and a shopping mall.

And in an international airport's arrival hall, it is more likely that individuals who flew in from the same airport of origin carries the same pathogen than any two random individuals who have just arrived. Putting samples of such individuals from the same airport of origin on the same input sampling plate will be more efficient than having them assigned to different plates.

As for the departure hall, it is more likely that individuals who stayed in the same hotel or lived at the same street have the same infectious disease than any two random individuals book a departing flight. Putting samples of such individuals from the same hotel or lived at the same street on the same input sampling plate will be more efficient than having them on different plates.

\section{Turnaround Time Is Not Pressing:}

Where the shortest shortaround time is not imperative, the Set 2 plate can wait be assembled till after the Set 1 plate has been fully tested to resolve which input sampling plate(s) is(are) positive. After the Set 1 test results are confirmed, only then the input sampling plate(s) that tested positive 
will contribute to the Set 2 pooled sample plate. This allows use of small volume microconcentrators and therefore fewer centrifuges, as well as bypasses the need for extra manpower to perform the 94 -to- 1 pooling across 94 plates and minimize the machine time on the 96-channel pipette so sharing the Set-1 96-channel pipette is possible.

\section{Where Cost/Availability of 96-Channel Pipette and/or Centrifuge Is An Issue:}

If the cost of the 96-channel pipette is prohibitive, or where there is a shortage, or where time to deliver equipment cannot meet an urgent need, the 96-channel pipette will have to be replaced by something less automated, perhaps

1. an 8- or 12-channel electronic pipette and a worker, or

2. two workers and two 12-channel electronic pipettes working on the same 96-well plate, facing each other, or

3. four workers and four 6-channel electronic pipettes each working on a different quadrant of the 96-well plate.

In this situation, cross contamination between wells must be avoided. Using a custom-made horizontal shield that slides/swings in and out over the wells during their movements can prevent any accidental drip or aerosolization from the pipette tips from falling into the wells.

The number of centrifuges can be reduced if not all microconcentrators from Set 1 are centrifuged at once. The total time to concentrate the sample pools grows inverse proportionately to the number of centrifuges.

Where Set 2 is processed after Set 1 results are finalized, either one or a handful of input sampling plates that test positive will be involved in Set 2 . Here, much smaller microconcentrator can be used, and many more of them can fit into the same centrifuge. And it can share the same centrifuge that is used for Set 1 .

\section{Livestock Farming:}

The MG can be used in livestock farming to detect the first sign of infection among livestock to guard against livestock disease such as the swine flu and avian flu. This can avoid mass destruction of an entire farm's livestock just to prevent spread of disease among the livestock, which is very expensive and takes long time to recover the business from.

Livestock that shares the same pen and eat from the same trough can have their samples pooled together and concentrated to form one sample to put into a well in the input sampling plate. And samples from pens that are proximal to each other and sharing the same air should be placed in the same input sampling plate because their holdings are more likely to cross infect each other than pens that are far away from each other or that are isolated from each other by walls. 


\section{VHT Multiplier Grid (MG) Drawings}

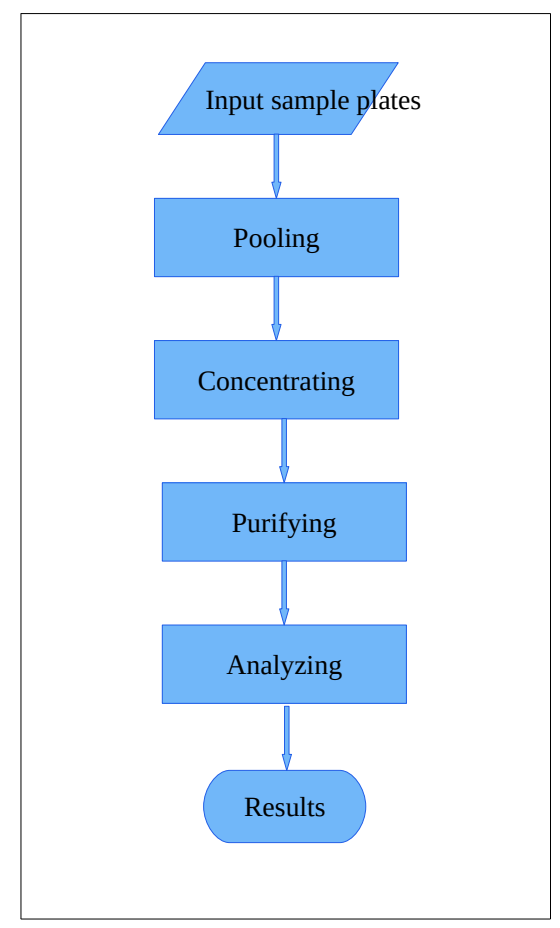

Figure 1. Flowchart: Input sample plates $\rightarrow$ pooling $\rightarrow$ concentrating $\rightarrow$ purifying $\rightarrow$ analysis.



Figure 2. Block diagram. 94 plates $\rightarrow$ Set 1 pooled plate + Set 2 pooled plate.

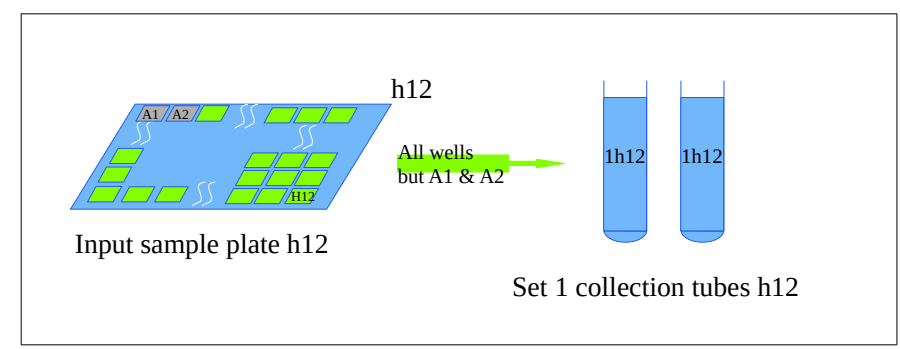

Figure 3. Block diagram. Input sample plate h12, all 94 wells $\rightarrow$ Set 1 collection tubes 1 h12. 


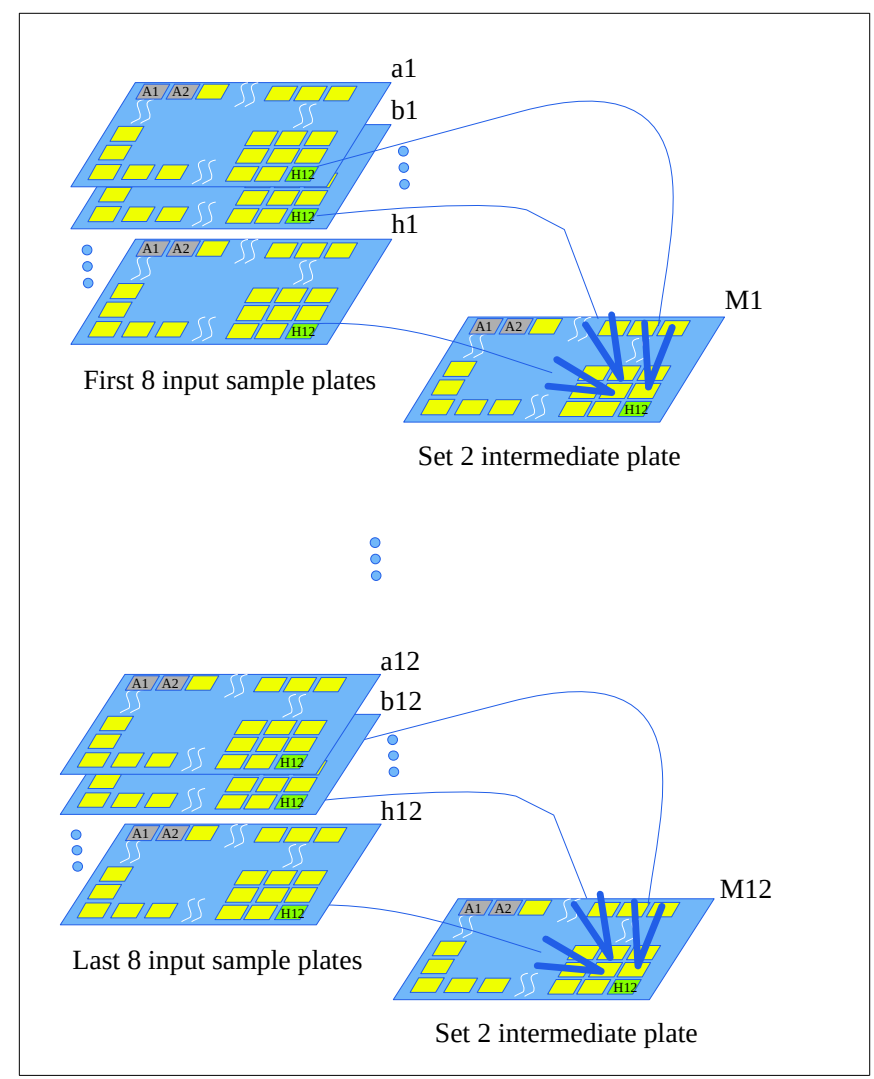

Figure 4A. Block diagram. Over 94 input sample plates, every 8 plates, all their A4 wells $\rightarrow$ one Set 2 intermediate pooling plate, well A4. Twelve(12) such intermediate pooling plates.

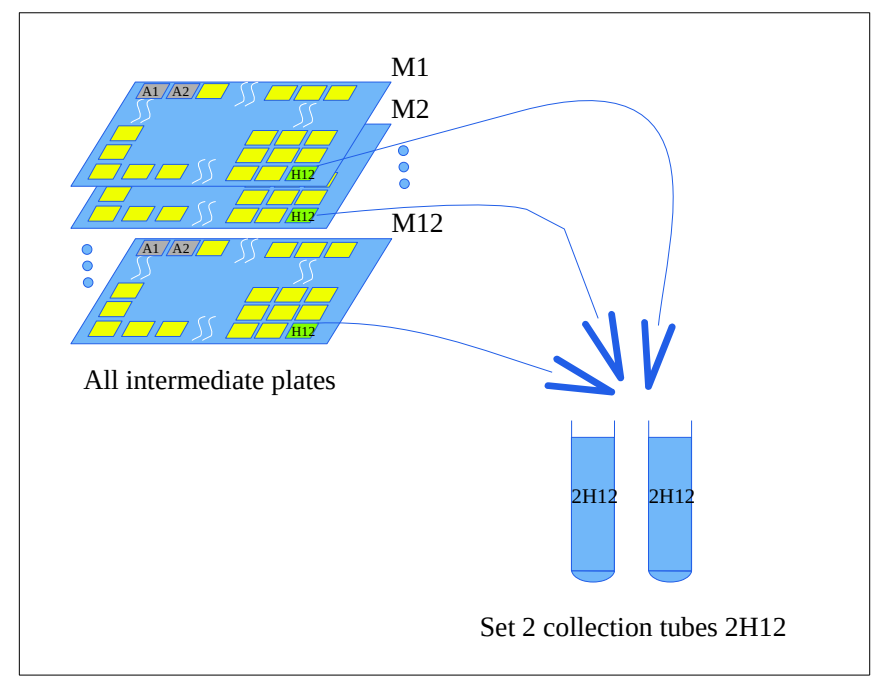

Figure 4B. Block diagram. All twelve(12) intermediate pooling plates M1 to M12, their H12 wells $\rightarrow$ Set 2 collection tubes $2 \mathrm{H} 12$. 


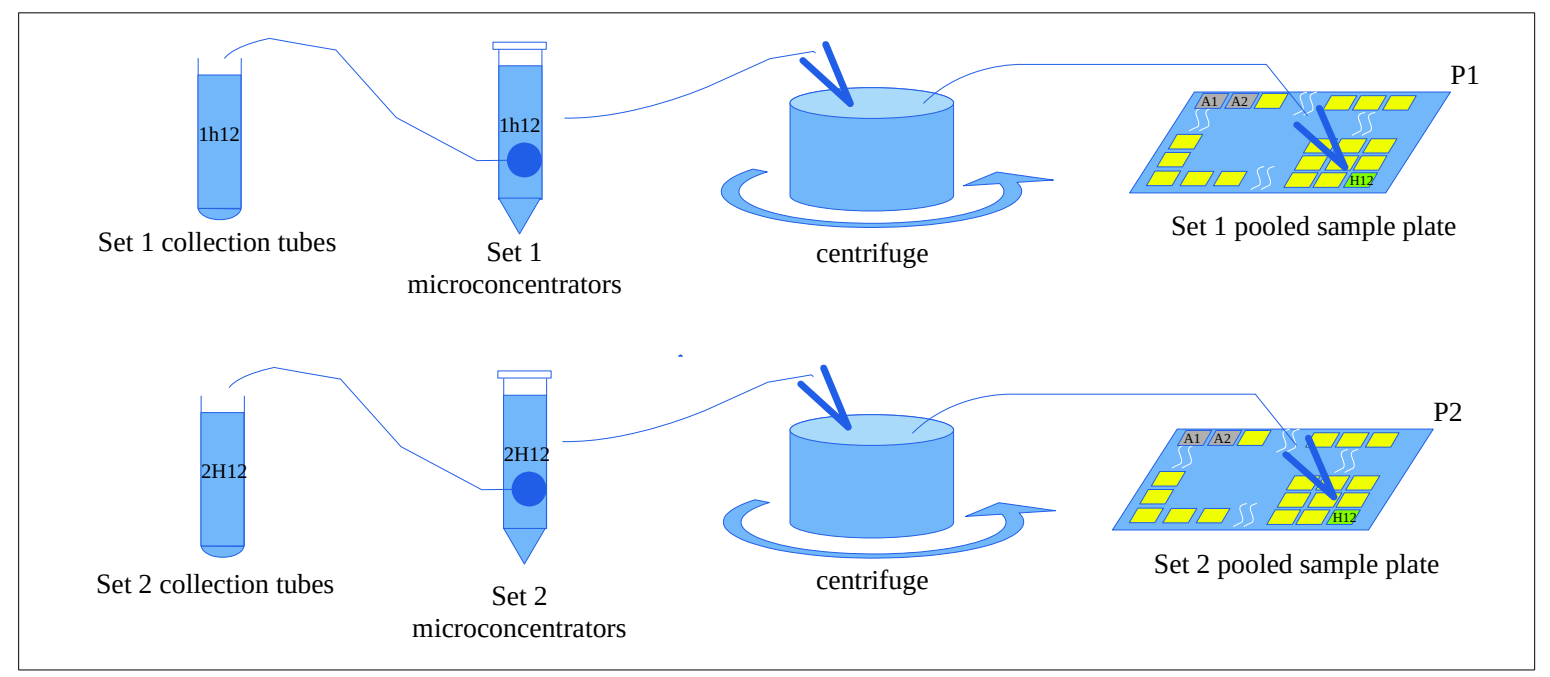

Figure 5. Block diagram. Set 1 Collection tube $1 \mathrm{~h} 12 \rightarrow$ microconcentrator $1 \mathrm{~h} 12 \rightarrow$ centrifuge $\rightarrow$ Set 1 pooled sample plate, well H12. And Set 2 collection tube $2 \mathrm{H} 12 \rightarrow$ microconcentrator $2 \mathrm{H} 12 \rightarrow$ centrifuge $\rightarrow$ Set 2 pooled sample plate, well H12.

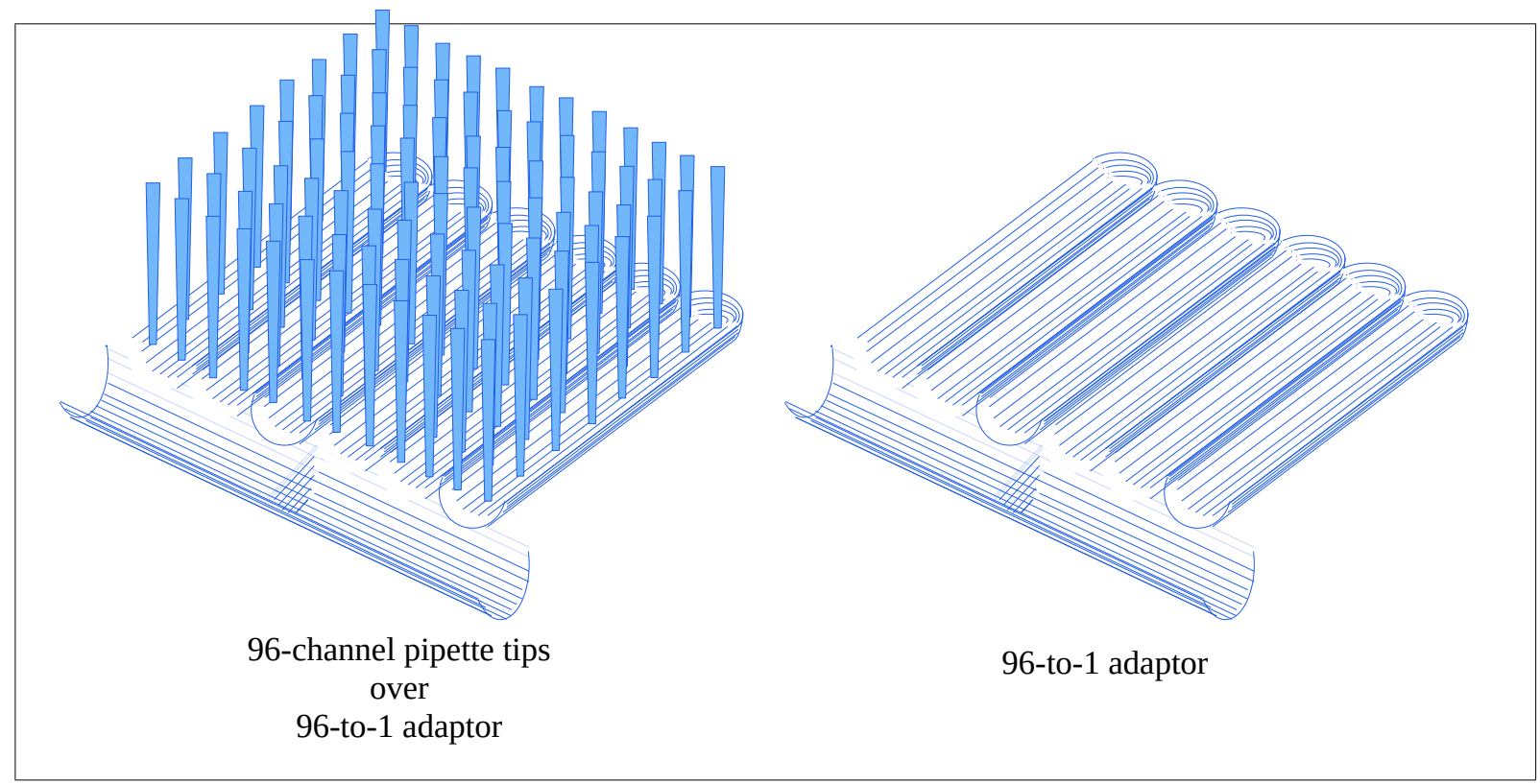

Figure 6. Perspective view of a 96-to-1 adaptor (right) and its alignment to 96-channel pipette tips (left). 